\title{
Numerical solution of two-dimensional Fredholm integral equations via modification of barycentric rational interpolation
}

\author{
Yubin Pan1, a, Jin Huang1, b \\ 1, a School of Mathematical Sciences, University of Electronic Science and Technology of China, \\ Chengdu, 611731, PR China \\ 1, b School of Mathematical Sciences, University of Electronic Science and Technology of China, \\ Chengdu, 611731, PR China \\ a email: yubinpan2014@163.com, bEmail: huangjin12345@163.com
}

Keywords: Barycentric Rational interpolation; Two-dimensional Fredholm integral equation of the second kind.

\begin{abstract}
We have presented a modified barycentric rational interpolation method for solving two-dimensional integral equations. The present method can accurately approximate the exact solution. We also compare with the Lagrange interpolation method and Nystöm method. The proposed method can achieve higher numerical accuracy than other two methods. At last, we give some examples to illustrate the validity of the presented method.
\end{abstract}

\section{Introduction}

Many scientific phenomena in science and engineering can be modeled by integral equations. Such as mathematical economics and optimal control theory [1, 2], boundary value problems of mathematics physics in [3]. There is a fact that many integral equations are usually difficult to solve analytically. So, it is important that the solutions of the integral equations can be computed by numerical methods.

There are many works on the study of numerical solutions of integral equations $[4,5]$. A modified interpolation method and Nystrom method have been introduced in [6, 7]. In [6], the author has presented an approach which is different and more general as well as more efficient than the Nystöm method, in the sense that Nystöm method becomes a particular case of the method developed here. Imran et al. in [8] developed the haar wavelet method for the numerical solutions of two-dimensional nonlinear integral equations. Nystöm method is a well-known method, and the method is easy to implement because we do not need to calculate any integral. But, Nystöm method has its own flaws, the given integral equation must be well behaved everywhere in the range of integration which ultimately helps in discretizing the given integral equation by utilizing standard quadrature rules. The present method is a modified barycentric rational interpolation method, the collocation nodes of traditional barycentric rational interpolation method are equidistant points or Gaussian nodes. In this paper, we give different method to discrete equations. Based on the barycentric rational interpolation with special collocation points, we will find that the order of accuracy can be improved greatly. From the numerical solution, we know the present method is good at solving two-dimensional Fredholm integral equations. We can conclude that the method is an efficient one and our work is meaningful.

\section{Barycenric Rational interpolation formula}

The barycentric rational interpolation basis can be expressed as follows: 
$r_{i}(x)=\frac{\sum_{j=0}^{n} \frac{w_{j}}{x-x_{j}} f_{j}}{\sum_{j=0}^{n} \frac{w_{j}}{x-x_{j}}}, \quad i=0,1, \cdots n$,

where $w_{j}=\sum_{i \in J_{k}}(-1)^{i} \prod_{k=i, k \neq j}^{i+d} \frac{1}{x_{j}-x_{k}}, J_{j}=\{i \in I: j-d \leq i \leq j\}$.

In this section, we introduce a method to solve two-dimensional Fredholm integral equations. We consider the following integral equations

$u(x, y)=\lambda \int_{a}^{b} \int_{c}^{d} k(x, y, s, t) u(s, t) d s d t+f(x, y)$

Where $u(x, y)$ is a known function, $\lambda$ is a constant, $k(x, y, s, t)$ and $f(x, y)$ are known function.

Now, we use the barycentric rational interpolation method to approximate the equation. The interval $[a, b]$ and $[c, d]$ are divided into equally spaced grid points $\left\{x_{k}\right\}_{k=0}^{n}$ and $\left\{y_{l}\right\}_{l=0}^{m}$ with step size $h_{n}=x_{k}-x_{k-1}$ and $h_{m}=x_{l}-x_{l-1}$. Let $x_{k, \alpha}=x_{k-1}+\alpha\left(x_{k}-x_{k-1}\right), y_{l, \alpha}=y_{l-1}+\alpha\left(y_{l}-y_{l-1}\right)$, $k=1,2, \cdots n$ and $l=1,2, \cdots m$. First, we approximate to unknown function as follows

$$
u(x, y)=\sum_{k=0}^{n} \sum_{l=0}^{m} u_{k, l} r_{k}(x) r_{l}(y)
$$

Where $u_{k, l}=u\left(x_{k}, y_{l}\right)$ represent unknown interpolation coefficient, $r_{k}(x)$ and $r_{l}(y)$ are basis functions of barycentric rational interpolation. Taking the above equation into (2), and then we obtain the following equation

$$
\sum_{k=0}^{n} \sum_{l=0}^{m} u_{k, l} r_{k}(x) r_{l}(y)=\lambda \sum_{k=0}^{n} \sum_{l=0}^{m} u_{k, l} \int_{a}^{b} \int_{c}^{d} k(x, y, s, t) r_{k}(s) r_{l}(t) d s d t+f(x, y) .
$$

We use Gauss-quadrature rule to calculate the integral of the right side of the above equation. Then (4) can be written as follows

$$
\sum_{k=0}^{n} \sum_{l=0}^{m} u_{k, l} r_{k}(x) r_{l}(y)=\lambda \sum_{k=0}^{n} \sum_{l=0}^{m} u_{k, l} \sum_{p=1}^{d} \sum_{q=1}^{d} c_{p} c_{q} k\left(x, y, s_{p}, t_{q}\right) r_{k}\left(s_{p}\right) r_{l}\left(t_{q}\right) d s d t+f(x, y),
$$

where $c_{p}, c_{q}, 1 \leq p, q \leq d$ are coefficients of Gauss-quadrature, and $s_{p}, t_{q}$ are Gauss-quadrature nodes . Replace the unknown $x, y$ in the above equation with $x=x_{k, \alpha}, y=y_{l, \alpha}$ and we can obtain the following algebra system

$$
\sum_{k=0}^{n} \sum_{l=0}^{m} u_{k, l} r_{k}\left(x_{k, \alpha}\right) r_{l}\left(y_{l, \alpha}\right)=\lambda \sum_{k=0}^{n} \sum_{l=0}^{m} u_{k, l} \sum_{p=1}^{d} \sum_{q=1}^{d} c_{p} c_{q} k\left(x_{k, \alpha}, y_{l, \alpha}, s_{p}, t_{q}\right) r_{k}\left(s_{p}\right) r_{l}\left(t_{q}\right) d s d t+f\left(x_{k, \alpha}, y_{l, \alpha}\right) .
$$

We need to add another equation

$$
\sum_{k=0}^{n} \sum_{l=0}^{m} u_{k, l} r_{k}\left(\gamma_{1}\right) r_{l}\left(\gamma_{2}\right)=\lambda \sum_{k=0}^{n} \sum_{l=0}^{m} u_{k, l} \sum_{p=1}^{d} \sum_{q=1}^{d} c_{p} c_{q} k\left(\gamma_{1}, \gamma_{2}, s_{p}, t_{q}\right) r_{k}\left(s_{p}\right) r_{l}\left(t_{q}\right) d s d t+f\left(\gamma_{1}, \gamma_{2}\right),
$$

By combining (6) and (7) together, we can obtain a linear system as follows:

$A u=b$,

Where $A$ is the coefficients matrix, $u=\left[u_{1,1}, u_{1,2}, \cdots, u_{1, n}, \cdots, u_{n+1, m+1}\right]$ are unknown terms. The

$u$ can be expressed as

$u=A^{-1} b$.

We give some examples to illustrate that the validity of the present method. We compare the present method with Nystrom method and the method in [7]. It should be noted that the well-known Nystrom method also requires a solution of a system of linear algebraic equations which can be derive form relation (6) by taking $\alpha=1$. 


\section{Examples}

In the section, we will give some examples to illustrate the validity of the present method. For each example use Gauss-quadrature rule to approximate the integral. For convenience, we consider the case of $m=n$.

Example1. We consider the following two-dimensional linear integral equation

$$
u(x, y)=x \cos (y)-\frac{1}{6}(\sin (1)+3) \sin (1)+\int_{0}^{1} \int_{0}^{1}(s \cdot \sin (t)+1) u(s, t) d s d t,
$$

Where the exact solution is $u(x, y)=x \cos (y)$.

We use the present method to approximate the exact solution of two-dimensional integral equation. We compare the absolution errors obtained by the present method and the method in [6] and the method in [7] for different ${ }^{n}$. Form the Tab.1, it is easy to find that when $n=6$ the method in [6] is better than the present method, but with the increase of $n$ the present method can reach a higher accuracy than the method in [6] and the method in [7]. In Fig. 1, we give the error map of some points which obtained by using the present method with $n=12$ and $d=n-1$. Form the figure, we can find that the errors of all the points are less than $2.5 \times 10^{-14}$. So, the numerical results show the superiority for solving two-dimensional Fredholm integral equation of the second kind.

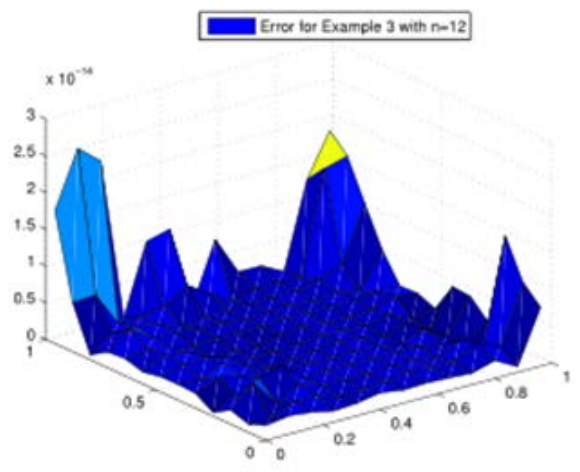

Fig1. The error of Ex. 1 with $n=12$

Table 1. The error for the Example 1 with different $n$.

\begin{tabular}{|c|c|c|c|c|c|c|c|c|}
\hline \multirow[t]{2}{*}{$(\mathrm{x}, \mathrm{y})$} & \multicolumn{2}{|l|}{$n=6$} & \multicolumn{2}{|l|}{$n=8$} & \multirow{2}{*}{$\begin{array}{c}n=10 \\
\text { ours }\end{array}$} & \multirow{2}{*}{$n=12$} & \multirow{2}{*}{$\frac{n=12}{[7]}$} & \multirow{2}{*}{$n=16$} \\
\hline & ours & [6] & ours & {$[6]$} & & & & \\
\hline$\left(\frac{1}{2}, \frac{1}{2}\right)$ & $4.54 \mathrm{e}-09$ & $5.399 \mathrm{e}-13$ & $4.89 \mathrm{e}-12$ & $4.22 \mathrm{e}-10$ & $8.88 \mathrm{e}-13$ & $2.83 \mathrm{e}-15$ & $9.5 e-06$ & $5.9 \mathrm{e}-07$ \\
\hline$\left(\frac{1}{4}, \frac{1}{4}\right)$ & $2.78 \mathrm{e}-09$ & $8.64 \mathrm{e}-10$ & $4.89 \mathrm{e}-12$ & $2.68 \mathrm{e}-10$ & $8.88 \mathrm{e}-13$ & $2.33 \mathrm{e}-15$ & $9.5 \mathrm{e}-06$ & $5.9 \mathrm{e}-07$ \\
\hline$\left(\frac{1}{8}, \frac{1}{8}\right)$ & $6.01 \mathrm{e}-09$ & $1.20 \mathrm{e}-09$ & $4.89 \mathrm{e}-12$ & $1.85 \mathrm{e}-10$ & $9.53 \mathrm{e}-13$ & $4.29 \mathrm{e}-15$ & $9.5 \mathrm{e}-06$ & $5.9 \mathrm{e}-07$ \\
\hline$\left(\frac{1}{16}, \frac{1}{16}\right)$ & $6.33 \mathrm{e}-09$ & $3.54 \mathrm{e}-10$ & $7.11 \mathrm{e}-12$ & $1.40 \mathrm{e}-10$ & $7.92 \mathrm{e}-13$ & $3.24 \mathrm{e}-15$ & $1.0 \mathrm{e}-05$ & $5.9 \mathrm{e}-07$ \\
\hline$\left(\frac{1}{32}, \frac{1}{32}\right)$ & $5.31 \mathrm{e}-09$ & $2.92 \mathrm{e}-10$ & $6.24 \mathrm{e}-12$ & $1.18 \mathrm{e}-10$ & $8.21 \mathrm{e}-13$ & $1.10 \mathrm{e}-14$ & $9.8 \mathrm{e}-06$ & $6.2 \mathrm{e}-07$ \\
\hline$\left(\frac{1}{64}, \frac{1}{64}\right)$ & $4.79 \mathrm{e}-09$ & $1.10 \mathrm{e}-10$ & $5.39 \mathrm{e}-12$ & $1.07 \mathrm{e}-10$ & $8.82 \mathrm{e}-13$ & $2.83 \mathrm{e}-15$ & $9.6 \mathrm{e}-06$ & $6.0 \mathrm{e}-07$ \\
\hline
\end{tabular}

Example2. Consider the following two-dimensional linear Fredholm integral equation

$$
u(x, y)=\frac{1}{1+x+y}-\frac{x}{1+y}+\int_{0}^{1} \int_{0}^{1} \frac{x(1+t+s)}{1+y} u(s, t) d s d t
$$

Where the exact solution is $u(x, y)=\frac{1}{1+x+y}$.

Tab.2 give the error results with some different $n$. Also, we compare the absolute error obtained by the present method and the method in [7]. From Tab.2, it is easy to find that the numerical results which obtained by using the present method is better than that obtained in [7]. In Fig.2, we give the error map which obtained by $n=16$ with $d=10$. From the figure, we can easy to find that when $n=16$ the errors are under the order of $10^{-11}$ magnitude. In Tab.3, we give the CPU time for Examples 1 and 2. From the results, we can find the present method spend significantly less time. 


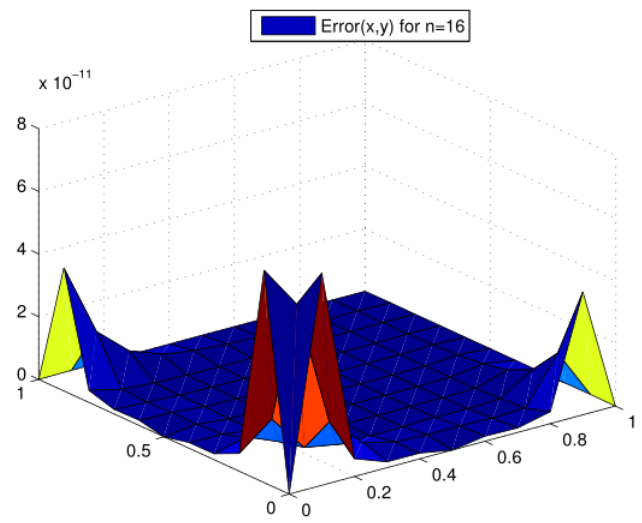

Fig2. The error of Ex. 2 with $n=16$

Table 2. The error for the Example 2 with different $n$

\begin{tabular}{cccccc}
\hline & $n=8$ & & & $n=16$ & \\
\cline { 5 - 6 } \cline { 5 - 6 } & ours & {$[7]$} & & ours & {$[7]$} \\
\hline$\left(\frac{0}{10}, \frac{0}{10}\right)$ & $0.00 \mathrm{e}-00$ & $0.00 \mathrm{e}-00$ & $0.00 \mathrm{e}-00$ & $0.00 \mathrm{e}-00$ \\
$\left(\frac{1}{10}, \frac{1}{10}\right)$ & $2.88 \mathrm{e}-07$ & $3.44 \mathrm{e}-04$ & $5.41 \mathrm{e}-11$ & $9.97 \mathrm{e}-05$ \\
$\left(\frac{2}{10}, \frac{2}{10}\right)$ & $7.08 \mathrm{e}-08$ & $4.63 \mathrm{e}-04$ & & $1.96 \mathrm{e}-12$ & $2.52 \mathrm{e}-05$ \\
$\left(\frac{3}{10}, \frac{3}{10}\right)$ & $1.70 \mathrm{e}-08$ & $2.01 \mathrm{e}-04$ & & $3.05 \mathrm{e}-13$ & $1.40 \mathrm{e}-05$ \\
$\left(\frac{4}{10}, \frac{4}{10}\right)$ & $3.64 \mathrm{e}-09$ & $7.48 \mathrm{e}-05$ & $1.49 \mathrm{e}-13$ & $1.68 \mathrm{e}-05$ \\
$\left(\frac{5}{10}, \frac{5}{10}\right)$ & $6.66 \mathrm{e}-16$ & $5.55 \mathrm{e}-17$ & & $3.11 \mathrm{e}-15$ & $3.33 \mathrm{e}-16$ \\
$\left(\frac{6}{10}, \frac{6}{10}\right)$ & $1.77 \mathrm{e}-09$ & $3.11 \mathrm{e}-05$ & & $2.94 \mathrm{e}-14$ & $8.43 \mathrm{e}-06$ \\
$\left(\frac{7}{10}, \frac{7}{10}\right)$ & $4.79 \mathrm{e}-09$ & $4.98 \mathrm{e}-05$ & & $1.28 \mathrm{e}-14$ & $2.87 \mathrm{e}-06$ \\
$\left(\frac{8}{10}, \frac{8}{10}\right)$ & $2.33 \mathrm{e}-09$ & $3.02 \mathrm{e}-05$ & & $3.19 \mathrm{e}-14$ & $2.02 \mathrm{e}-06$ \\
$\left(\frac{9}{10}, \frac{9}{10}\right)$ & $2.87 \mathrm{e}-09$ & $1.26 \mathrm{e}-05$ & & $1.67 \mathrm{e}-13$ & $2.93 \mathrm{e}-06$ \\
$\left(\frac{10}{10}, \frac{10}{10}\right)$ & $1.33 \mathrm{e}-15$ & $1.67 \mathrm{e}-16$ & & $5.55 \mathrm{e}-15$ & $0.00 \mathrm{e}-00$ \\
\hline
\end{tabular}

Table 3. The CPU time (in seconds)

for the Examples 1 and 2 with different $n$.

\begin{tabular}{ccccc}
\hline Ex & $n=6$ & $n=8$ & $n=10$ & $n=12$ \\
\hline Ex1 & 0.347 & 0.510 & 0.833 & 1.441 \\
Ex2 & 0.206 & 0.391 & 0.703 & 1.238 \\
\hline
\end{tabular}

\section{Conclusion}

In this paper, we give a modified barycentric rational interpolation method for solving two-dimensional Fredholm integral equations of the second kind. For solving two-dimensional integral equations, we let $\alpha=0$ and $\gamma=1$ with some suitable $d$. By using barycentric rational interpolation method, a better numerical solution can be obtained. Form the numerical result we can conclude that the modified barycentric rational interpolation method is a valid method for solving two-dimensional Fredholm integral equations of the second kind. Next, we consider solving some more practical problems.

\section{Acknowledgement}

In this paper, the research was sponsored by the National Natural Science Foundation of China (11371079). 


\section{References}

[1] Bazm S. Bernoulli polynomials for the numerical solution of some classes of linear and nonlinear integral equations [J]. Journal of Computational \& Applied Mathematics, 2015, 275:44-60.

[2] Wazwaz A.M. Linear and Nonlinear Integral Equations: Methods and Applications [M].Higher Education Press, 2011.

[3] Williams W E. Note on the scattering of water waves by a vertical barrier [J].Mathematical Proceedings of the Cambridge Philosophical Society, 1966, 62(3):507-509.

[4] Müller F, Varnhorn W. On approximation and numerical solution of Fredholm integral equations of second kind using quasi-interpolation [J].Applied Mathematics and Computation, 2011, 217(13):6409-6416.

[5] Okayama T, Matsuo T, Sugihara M. Improvement of a Sinc-collocation method for Fredholm integral equations of the second kind [J]. BIT, 2011, 51(2):339-366.

[6] Panda S, Martha S C, Chakrabarti A. A modified approach to numerical solution of Fredholm integral equations of the second kind [J], Applied Mathematics and Computation, 2015, 271(C):102-112.

[7] Atkinson E.K.The numerical solutions of integral equations of the second kind.Cambridge University Press, 1997 (4).

[8] Aziz I, Siraj-ul-Islam, Khan F. A new method based on Haar wavelet for the numerical solution of two-dimensional nonlinear integral equations[J]. Journal of Computational and Applied Mathematics, 2014, 272(272):70-80. 